\title{
Rare Presentation of Benign Prostatic Cyst: A Case Report
}

\author{
Noopur Bansal ${ }^{1}$, Amrik S Bhatia ${ }^{2}$, Abhinav Mahajan ${ }^{3}$
}

\begin{abstract}
Background: Prostatic cyst is a rare disease of prostate, with $0.5-7.9 \%$ prevalence. It is often asymptomatic and found accidentally using abdominal ultrasound, computed tomography (CT), or magnetic resonance imaging (MRI). It is a rare pathology which can be confused with diseases of prostate like benign hyperplasia of prostate or carcinoma prostate which are much more common in old age.

Case description: A 70-year-old male presented to emergency department with complaints of acute urinary retention and constipation. Abdomen was opened via a lower midline incision revealing a large cystic lesion of prostate which was aspirated (550 mL approximately, brownish in color) and deroofing of cyst wall was done. Complete excision could not be done due to the presence of adhesion with rectal wall. Histopathology report confirmed it to be a benign cyst of prostate.

Discussion: Existing literature of prostatic cysts are highlighting its infrequent occurrence. The etiological factors of prostatic cyst include inflammatory disease, benign prostatic hyperplasia, ejaculatory duct obstruction, atrophy of prostate gland, and tumor. In our case, the patient had urinary retention and constipation which was related to large size of the cyst. The differential diagnosis includes bladder diverticulum, teratoma, seminal vesicle cyst, epididymal cyst, and Wolffian duct cyst.

Keywords: Benign hyperplasia prostate, Carcinoma prostate, Prostatic cyst.

AMEl's Current Trends in Diagnosis \& Treatment (2019): 10.5005/jp-journals-10055-0070
\end{abstract}

\section{INTRODUCTION}

Prostatic cyst is a rare disease of prostate with $0.5-7.9 \%$ prevalence. ${ }^{1}$ It is often asymptomatic and found accidentally with abdominal ultrasound, computed tomography (CT), or magnetic resonance imaging (MRI). It is a rare pathology which can be confused with diseases of prostate like benign hyperplasia of prostate or carcinoma prostate which are much more common in old age.

\section{Case Description}

A 70-year-old male presented to the emergency department with complaints of acute urinary retention and constipation. He had long-standing symptoms of bladder outlet obstruction and repeated catheterization with complaint of constipation. Abdominal examination was normal. Rectal examination revealed a nontender smooth cystic swelling anteriorly from which prostate could not be separately delineated. It was not possible to get above the swelling. Rectal mucosa was normal and freely mobile.

Abdominal ultrasonography revealed cystic swelling in close relation to prostate and rectal wall of approximate $9 \times 8 \times 8 \mathrm{~cm}$ in size. Serum prostate-specific antigen (PSA) levels increased to 229 $\mathrm{ng} / \mathrm{mL}$ while the cytology of transrectal ultrasonography-guided

\footnotetext{
1,2Department of General Surgery, Sri Guru Ram Das Institute of Medical Sciences and Research, Amritsar, Punjab, India

${ }^{3}$ Department of General Surgery, Government Medical College, Patiala, India
}

Corresponding Author: Noopur Bansal, Department of General Surgery, Sri Guru Ram Das Institute of Medical Sciences and Research, Amritsar, Punjab, India, Phone: +91 7707808599, e-mail: nbansal000@ gmail.com

How to cite this article: Bansal N, Bhatia AS, Mahajan A. Rare Presentation of Benign Prostatic Cyst: A Case Report. AMEl's Curr Trends Diagn Treat 2019;3(2):82-83.

Source of support: Nil

Conflict of interest: None

aspirated fluid (350 $\mathrm{mL}$, brownish colored) came out to be nondiagnostic. Patient was planned for CT scan which confirmed ultrasound findings of large well-defined, rounded thin-walled predominantly cystic lesion in rectoprostatic space approximately $8.9 \times 8.4 \times 8.1 \mathrm{~cm}$ in size with fluid level within it (Fig. 1). Anteriorly, it was abutting posterior part of prostate on right side and right

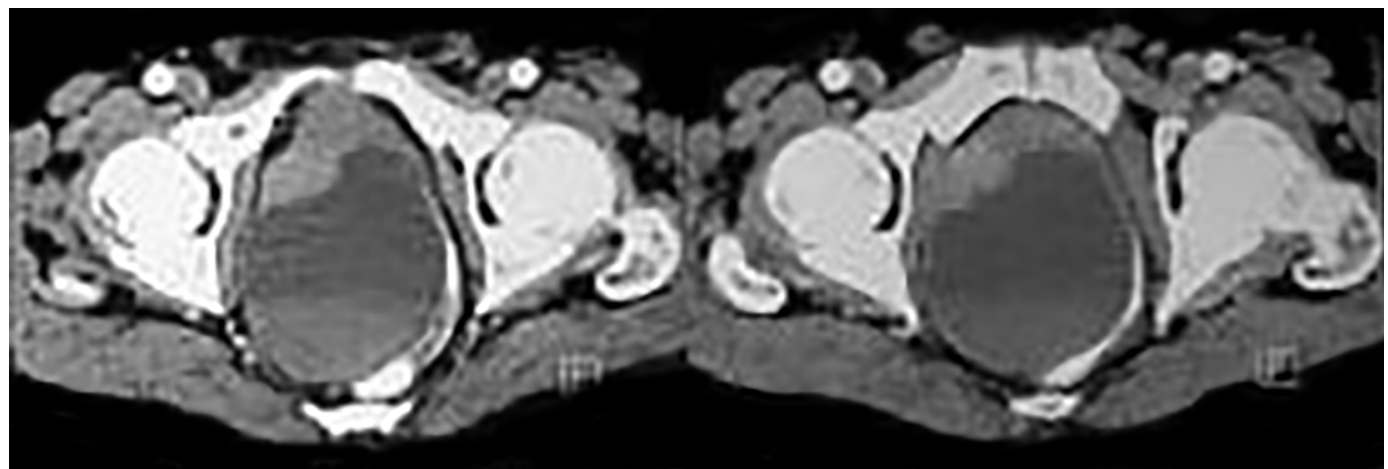

Fig. 1: Contrast-enhanced computed tomography showing large cystic lesion

C) The Author(s). 2019 Open Access This article is distributed under the terms of the Creative Commons Attribution 4.0 International License (https://creativecommons. org/licenses/by-nc/4.0/), which permits unrestricted use, distribution, and non-commercial reproduction in any medium, provided you give appropriate credit to the original author(s) and the source, provide a link to the Creative Commons license, and indicate if changes were made. The Creative Commons Public Domain Dedication waiver (http://creativecommons.org/publicdomain/zero/1.0/) applies to the data made available in this article, unless otherwise stated. 


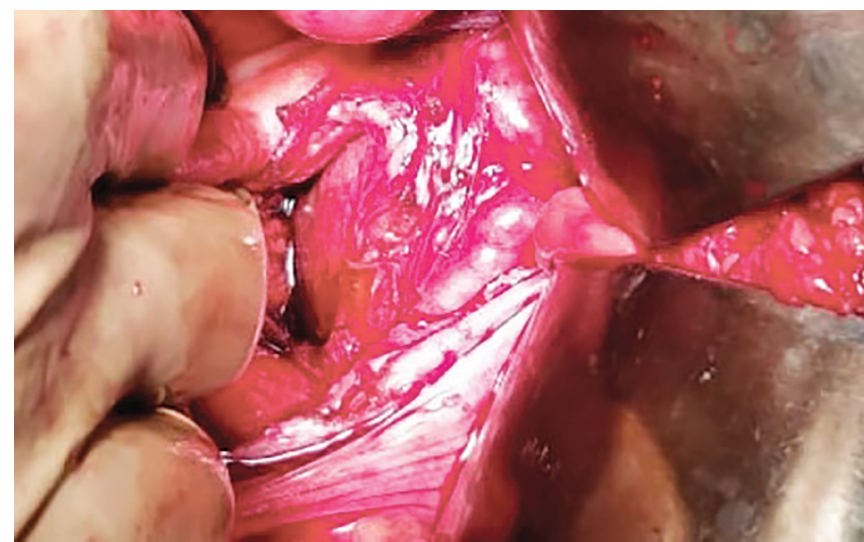

Fig. 2: Intraoperative picture showing prostatic cyst

obturator internus muscle with ill-defined fat planes. Posteriorly, cystic lesion was compressing on rectum and displacing it posterolaterally, its fat planes from anterior wall of rectum were not well defined. Inferiorly, lesion was reaching up to pelvic diaphragm. Superiorly it was reaching opposite to S2 vertebral body.

Abdomen was opened via a lower midline incision revealing a large cystic lesion of prostate which was aspirated (approximately $550 \mathrm{~mL}$ and brownish) and deroofing of the cyst wall was done (Fig. 2). Complete excision could not be done due to the adhesion with rectal wall. Postoperative period was uneventful. Histopathology report confirmed it to be a benign cyst of prostate.

\section{Discussion}

Existing literature of prostatic cysts are highlighting its infrequent occurrence. According to Mou et al., ${ }^{2}$ the etiological factors of prostatic cyst include inflammatory disease, benign prostatic hyperplasia, ejaculatory duct obstruction, ${ }^{3}$ atrophy of prostate gland, and tumor. Clinical manifestations depend on the size of cyst and vary from asymptomatic to recurrent urinary tract infections, epididymitis, hematuria, pyuria, urinary incontinence, oligospermia, lower abdominal discomfort/heaviness, urinary retention or constipation, bladder outlet obstruction, perineal pain, hematospermia, painful ejaculation, ${ }^{4}$ irritative and/or obstructive lower urinary tract symptoms, infertility, ${ }^{5}$ and dysuria. In our case, the patient had urinary retention and constipation which was related to the large size of the cyst. The differential diagnosis includes bladder diverticulum, teratoma, seminal vesicle cyst, epididymal cyst, and Wolffian duct cyst. At the present time, therapeutic options consist of transurethral resection, transrectal ultrasound-guided aspiration with or without sclerotherapy, and open surgery.

\section{Conclusion}

Symptomatic prostatic cysts are very rare and they need to be differentiated from the more common causes of prostatic symptoms.

As some large prostatic cysts would have high serum PSA, it should be differentiated from prostatic neoplasm.

Prostatic cyst with progressive symptoms, large size $(2.5 \mathrm{~cm}$ or larger), or high serum PSA should be timely managed.

\section{References}

1. Galosi AB, Montironi R, Fabiani A, et al. Cystic lesions of the prostate gland: an ultrasound classification with pathological correlation. J Urol 2009;181(2):647-657. DOI: 10.1016/j.juro.2008.10.006.

2. Mou P, Zijun W, Mao D, et al. Giant multilocular prostatic cysts treated by laparoscopic prostatectomy: a rare case report in china mainland. Int J Clin Exp Med 2016;9:13227-13230.

3. Jarow JP. Diagnosis and management of ejaculatory duct obstruction. Tech Urol 1996;2(2):79-85.

4. Cheng G, Liu B, Song Z, et al. A novel surgical management for male infertility secondary to midline prostatic cyst. BMC Urol 2015;15(1):18. DOI: 10.1186/s12894-015-0015-8.

5. Issa MM, Kalish J, Petros JA. Clinical features and management of anterior intra urethral prostatic cyst. Urology 1999;54(5):923. DOI: 10.1016/s0090-4295(99)00315-5. 